\title{
Improving Search on WWW.HR Web Directory by Introducing Ontologies
}

\author{
Gordan Gledec, Maja Matijašević, Damir Jurić \\ Faculty of Electrical Engineering and Computing \\ University of Zagreb, Croatia \\ \{maja.matijasevic, gordan.gledec, damir.juric\}@fer.hr
}

\begin{abstract}
In this paper we propose ontology-based improvement of the Croatian Web directory search mechanism, which is currently not capable of executing queries that take into account the structure and semantics of user's query. The proposed approach is verified by introducing an ontology in the domain (directory category) of "tourism". We address three problems related to the search mechanism: low recall, high recall and low precision and vocabulary mismatch. The results show significant improvements in terms of subjective relevance and quality of results.
\end{abstract}

\section{Introduction}

The directory of Croatian Web servers, named WWW.HR, was first introduced a decade ago [1]. As the directory grew in terms of both the number and variety of sites included, it became increasingly difficult to find the information needed, especially for inexperienced users not familiar with formulating logical queries.

Recent study of user queries [2] on Croatian Web directory identified several problems related to the number and relevance of search results. These problems clearly indicate the need for new search mechanisms that will enable semantic search on the directory. In this paper, we address these problems by introducing ontology-based improvements into the search engine.

In the last few years, with the introduction of Semantic Web [3], a lot of research is under way in the field of ontology-based search [4], [5], [6]. Some visionary ideas on how Web search will evolve toward question answering systems can be found in [7].

Our research is inspired by Navigli and Velardi [4], who show that introducing other types of semantic information derivable from an ontology into the Web site search mechanism is effective at improving search results. To the best of our knowledge, this is the first study that analyses benefits of ontology-based search improvements related to the Croatian language and the Croatian Web space, however, the approach and method applied in this work are rather general and not languagespecific.

The remainder of this paper is organised as follows. Section 2 explains the structure of Croatian Web directory and current search mechanisms. Section 3 describes 
the idea of ontology-based directory search. Section 4 explains how the ontology was implemented. Section 5 presents the results. Finally, Section 6 concludes the paper and gives references for future work.

\section{WWW.HR directory and search}

The WWW.HR is a Web-based information service supported by the Croatian Academic and Research Network - CARNet. Established in 1994, WWW.HR tends to be a thematic portal, providing general information concerning Croatia and a directory of Croatian Web sites. The directory is organized into a hierarchical structure similar to Yahoo (http://www.yahoo.com/) or DMOZ (http://www.dmoz.org/), starting from broad, more general categories, to narrower, more specific subjects, leading finally to a Web page that (presumably) has information on the subject. The top level of directory contains 14 categories, namely: About Croatia, Arts \& Culture, Business \& Economy, Computers \& Networking, Education, Entertainment, Events, Law \& Politics, News \& Media, Organizations, Science \& Research, Sport \& Recreation, Society, and, Tourism \& Traveling.

The directory is updated and expanded based on site submissions. New sites are submitted through Web interface. The following information about each site is recorded in the directory database and used by the master search index file:

- site name (in English and Croatian),

- site description (in English and Croatian),

- site URL,

- category name in which the site is submitted (in English and Croatian).

The master search index file also contains META keywords and description extracted from the Web page headers. When the user enters the query into the search form, the search index is queried and matching results returned to the user. The types of user queries supported are:

- all keywords entered (logical and between the terms, this is the default query),

- logical expressions (using and, or, not, + and - operators),

- keywords ending with wildcards (an asterisk representing any keyword suffix),

- phrases (any character string in quotation marks).

In order to gain knowledge of how users search our directory, we conducted the study of user queries which revealed that more than $60 \%$ of queries consisted of a single term, typically resulting in a rather large number $(>20)$ matches. The type of query terms analysis revealed that $70 \%$ of all terms were nouns. Other problems discovered in our study may be summarized as:

(1) no recall: almost $30 \%$ of all queries returned no relevant results;

(2) low precision and high recall: almost $40 \%$ of queries returned 15 or more results;

(3) vocabulary mismatch: results of the query highly depend on the vocabulary (particular terms) the users use in formulating their query. 
If a user enters a search term which is not used within either the title, or the description or the META tag(s) the query returns no matching results. If the query is too general, this results in high recall, so the user still has to scroll through a large amount of results to find the relevant one.

To improve the search in terms of semantics, we first had to build an ontology. We selected one of the top-level categories, namely "Tourism", as a case study, since Web log statistics show that this directory was most frequently accessed, either by browsing or searching.

\section{Idea of ontology-based directory search}

In "classical" search, the user enters the query term(s), and receives result(s) as a list of matching directory entries (Fig. 2, steps 1-2-9-10). The idea of enhancing this with the ontology-based search is illustrated in Fig. 2, steps 1 through 10. The user enters the query (1) into the search form, and submits it to the search engine (2). The engine consults the ontology (3) and extracts the relevant knowledge (4). The list of terms is extracted from the ontology and presented to the user (5) who can select the relevant terms (6) he wants to include in the modified query (7). Upon the submission of the modified query (8), the search engine retrieves results from the search index (9) and presented to the user (10).

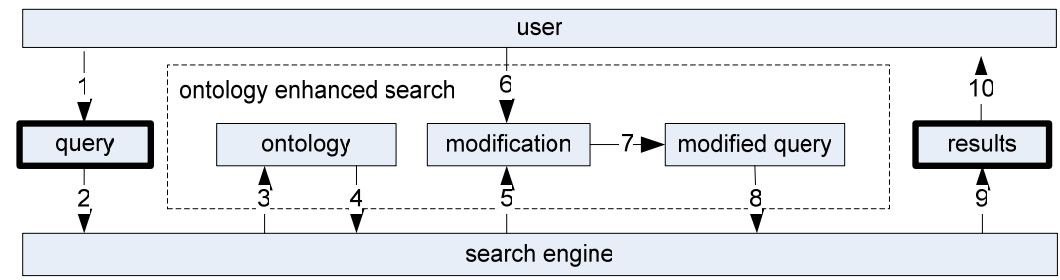

Fig. 1. The process of query modification using ontologies

In order to implement this solution, the ontology has to be built. Since the directory is rather general, and the user is a public user, this directory would be covered by many domain-related ontologies. The knowledge of user behaviour and expectations, gained from the study described in Section 2, was used as a guidance.

Building the ontology is the most difficult and time-consuming task, since no automated tools are used and the process involves only human knowledge. As such, the process of ontology creation can be split into three phases:

(a) domain consideration,

(b) planning the domain,

(c) coding the ontology.

Domain consideration involves decisions like what should be included as a part of the ontology and what should be omitted. Planning the domain requires consultation with relevant sources of information. In the case of directory based search, the 
already existing categorization and subcategorisation in the directory, as well as the analysis of the most frequently used keywords used to search the directory may be used. Still, certain parts of knowledge must come from the ontology creator, whose role is to synthesize knowledge from different sources, which is still possible until ontologies become rather small and domain specific. Coding the ontology in a tehnicaly acceptable format is the last step in the process of creating the ontology.

\section{Implementation}

Our ontology was created taking into account the existing categorization of the "Tourism" section and keywords that users use while searching the directory. The knowledge defined in the ontology is represented by subclasses of the query term, alternative terms or synonyms, upclasses and possible restrictions.

In the domain consideration phase we focus only on important concepts which are usualy described in terms of nouns. Many of these already exist as a part of the directory categories. The top-level directory of „Tourism" comprises of 14 categories with aditional subcategories under each of them: Accommodation, Food and Beverage, Cultural and Historical Monuments, Agencies and Associations, Gastronomy, Traveling and Transport, Guides, Web Cameras, Islands, Yachting, Nature, Spas, Places and Regions.

In the phase of planning the domain, statistics obtained from the Web server access logs were used to decide which concepts to include in the ontology. In our study, most of the main classes within the ontology were extracted from already existing categorisation in the directory. One superclass was created ("tourist"), and related to other classes by relations such as "to see", "to offer", "to give information about" or "located in", as shown in Fig. 1. For ontology coding, we used the W3C Ontology Web Language [9].

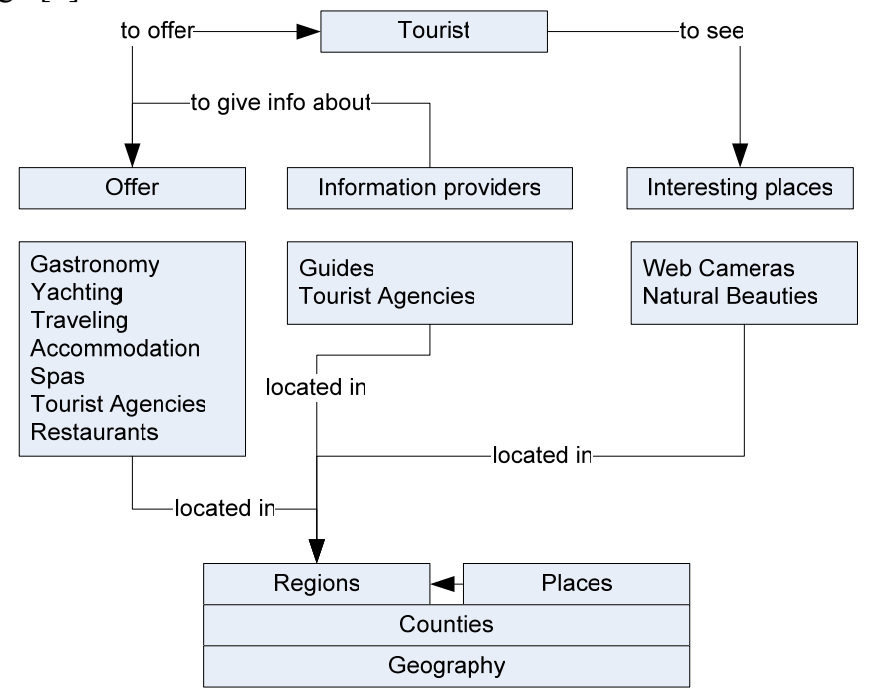

Fig. 2. Ontology "tourism" 
This enhancement to the "classical" search was implemented by a set of custom made perl scripts.

Fig. 3. shows the user interface of the enhanced search page, containing the form presented to the user in step (5) on Fig. 2. The form includes options for the modifying the query by selecting alternative terms and synonyms.

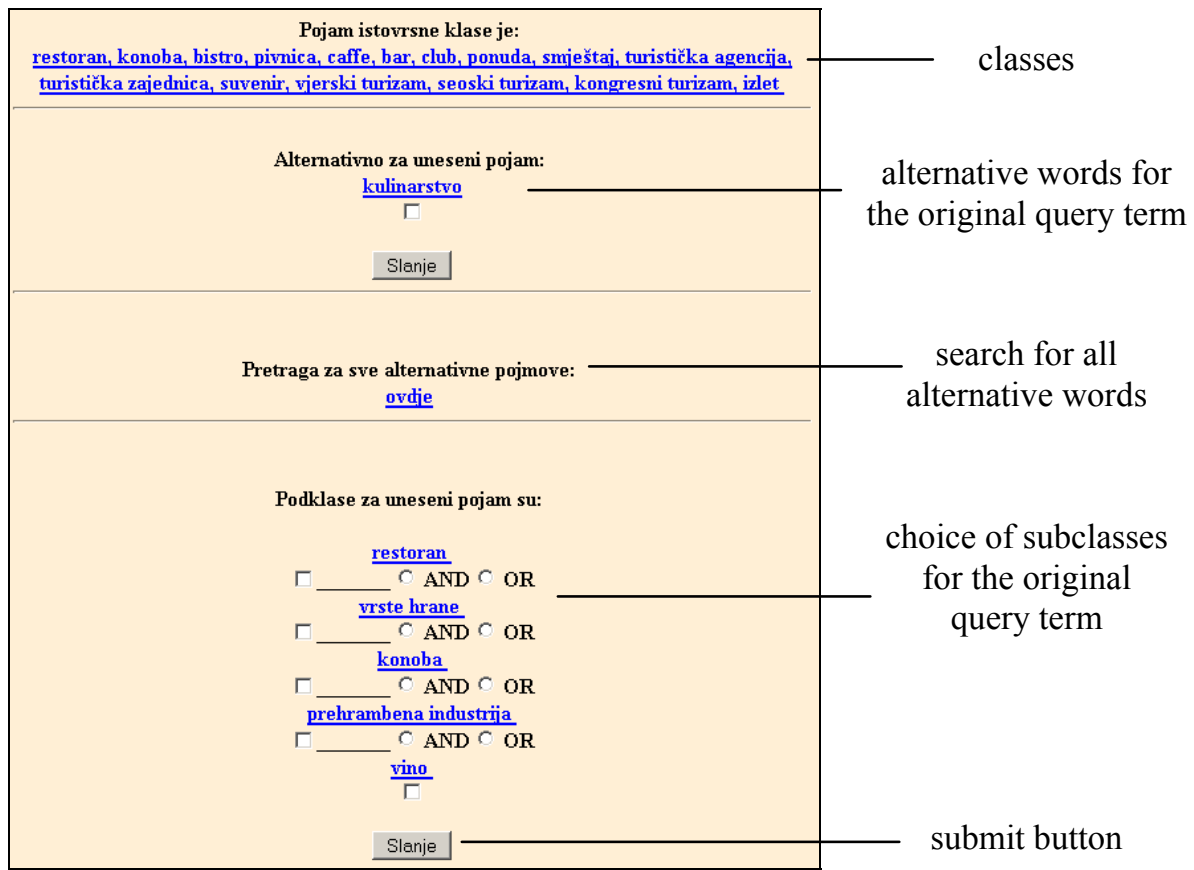

Fig. 3. The form which enables the user to modify the query

\section{Results}

We addressed the problems associated with search mechanisms, low recall, high recall and low precision and vocabulary mismatch by conducting three types of tests.

The first test was designed to address the problem of low recall. The idea to approach this problem was to modify the original user query to include all subclasses of the query term. When the query was modified with all subclasses included, the search obviously retrieved more results than the original search. For example, searching for query prirodne ljepote (natural beauties) retrieved 43 results, but with subclasses like nacionalni parkovi (national parks) and rezervat (reservation) included, it retrieved 76 results (Fig. 4 -1a). Another example is autprijevoz (car transport). The search for this term retrieves only 3 results, but when subclasses like autobusna agencija (bus agency), rent-a-car, autobusni prijevoz (bus transport), 
kamionski prijevoz (truck transport) and similar are included in the search,. the search engine returns 56 results (Fig. $4-1 \mathrm{~b}$ ).

The second test aimed at addressing the problem of high recall and low precision based on restricting the original query. Searching with restrictions resulted in less matches because of specific requirements (eg. location), so only pages matching these requirements were taken into account. An example is the query for the term aerodrom (airport), which retrieved 9 results, but the ontology found restriction for location and offered a choice of cities (Zagreb, Zadar, Split) to the user, so the number of matching results can be much smaller (Fig. $4-2 b$ ). Another example is the search for the term apartman (appartment) - the search engine returns hundreds of results, but with restrictions that include the keyword kuhinja (kitchen) the number of retrieved results is restricted to managable 11 (Fig. $4-2 b$ ).

The third test addressed the problem of vocabulary mismatch by modifying the original query by including synonyms and alternative words extracted from the ontology. Croatian language is very rich in synonyms and dialects. When synonims or alternative words were included in our ontology, the search retrieved more relevant results than the original query, as expected. For example, the word "car" has several forms in Croatian language: automobil (automobile), auto (auto), vozilo (vehicle), to name just a few. The search returned 43 results for the keyword automobil. When the query was modified to include all three forms, the number of retrieved results was 100 (Fig. 4 - 1a). The query for the word dvorac (castle) returned 11 results, but when it was modified with words that have similar meanings - kula (tower), palača (palace), zamak (court), utvrda (fortress), dvor (court) - the number of matching results grew to 41 when all keywords were selected for the final query (Fig. $4-3 b$ ).

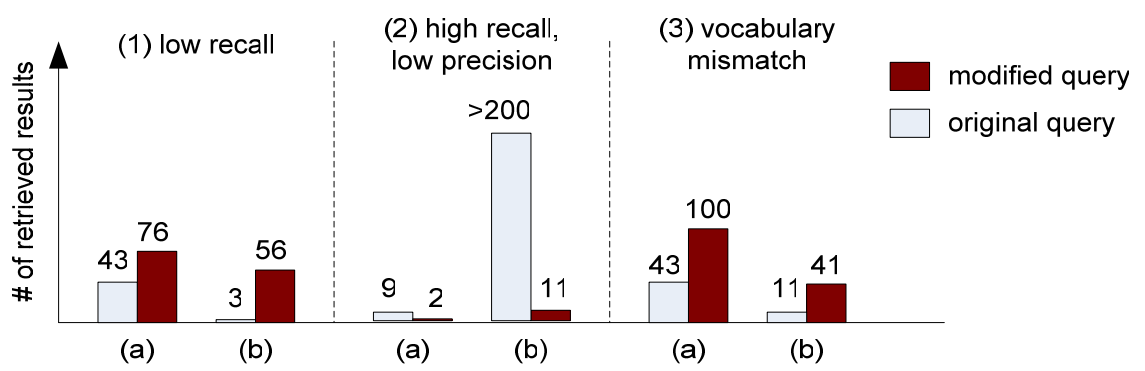

Fig. 4. The comparison between "classical" (string-based) and ontology-based search

\section{Conclusions and future work}

The results may be summarized as follows. The query modifications that involved the inclusion of synonyms and subclasses returned many more relevant results than the original search, and the modification with restrictions returned fewer of results, but with greater relevance. (The relevance of the retrieved results throughout this paper was assessed subjectively.) 
It may also be noted that combining the proposed methods of query modifications may increase search efficiency. The query can be modified by first expanding it with the synonyms and then restricting it to give more focused results. For example, if the original query searches for the term aerodrom (airport), it could first be expanded by adding synonym zracna luka, and then restriction to a preferable location, for example Split, could be applied to narrow the number of matching results.

Our future work will focus on developing ontologies for other frequently accessed parts of the directory. Also, we need to improve a rather rudimentary user interface to enable users to modify their queries in a more usable fashion. Additionally, we need to perform a more formal user survey to assess the relevance of results retrieved through ontology-based search.

\section{References}

1. Jurić, J., Matijašević, M., Mikuc, M. First experiences with the World-Wide-Web in Croatia, Proceedings of the 2nd International Conference on Telecommunication Information Systems, TIS '94, pp. 90-95, Žilina, Slovakia (1994)

2. Gledec, G., Ljubi, I. How Users Search WWW.HR Web Directory? Proceedings of the 6th CARNet Users Conference, CD-ROM edition. Zagreb (2004)

3. Berners-Lee, T., Hendler, J., Lassila, O. The Semantic Web, Scientific American, vol. 284, No. 5. pp. 34-43 (2001)

4. Navigli, R., Velardi, P. An Analysis of Ontology-based Query Expansion Strategies, Proceedings of the 14th European Conference on Machine Learning (ECML 2003), CavtatDubrovnik, Croatia (2003)

5. Ding, L., Finin, T., Joshi, A., Peng, Y., Scott Cost, R., Sachs, J., Pan, R., Reddivari, P., Doshi, V. Swoogle: A Semantic Web Search and Metadata Engine, Proceedings of the $13^{\text {th }}$ ACM Conference on Information and Knowledge Management (CIKM'04), Washington DC (2004)

6. Modica, G., Gal, A., Jamil, H. M.; The Use of Machine-Generated Ontologies in Dynamic Information Seeking, Proceedings of the 6th International Conference on Cooperative Information Systems (CoopIS 2001), Springer-Verlag LNCS series, September 5-7, Trento, Italy (2001)

7. Zadeh, A.L.: Web Intelligence, World Knowledge and Fuzzy Logic - The Concept of Web IQ (WIQ), Proceedings of the $8^{\text {th }}$ International Conference KES 2004, Wellington, New Zealand (2004)

8. Antoniou, G., van Harmelen F.: A Semantic Web Primer. The MIT Press (2004)

9. OWL - Web Ontology Language Reference, URL: http://www.w3.org/TR/owl-ref/ (2004) 